\title{
An online short-term bed occupancy rate prediction procedure based on discrete event simulation
}

\author{
Zhu Zhecheng \\ Health Services and Outcomes Research (HSOR) in National Healthcare Group (NHG), Singapore
}

Correspondence: Zhu Zhecheng. Address: \#04-01, 6 Commonwealth Lane, GMTI Building, Singapore 149547. E-mail: zhecheng_zhu@nhg.com.sg

Received: October 28, 2013

Accepted: January 22, 2014

Online Published: February 19, 2014

DOI : $10.5430 /$ jha.v3n4p37

URL: http://dx.doi.org/10.5430/jha.v3n4p37

\begin{abstract}
In recent years, population growth and aging society impose large pressure on the resource requirement in Singapore public hospital system. Beds are one of the most critical resources in healthcare system. How to manage beds efficiently is an important and challenging task for the health service providers in any healthcare systems. One frequently used performance indicator of bed management is bed occupancy rate, which measures the bed utilization. In this paper, an online prediction procedure based on discrete event simulation is proposed and developed to predict bed occupancy rate in a short term period. Simulation results show that the predicted values are closer to the actual values with narrower confidence interval compared to the offline approach. Hence such a prediction procedure is able to provide a more reliable reference for decision making of the health service providers.
\end{abstract}

\section{Key words}

Bed occupancy rate, Discrete event simulation, Prediction

\section{I ntroduction}

One common challenge most hospitals are facing is how to cope with the increasing patient load with the limited resources due to population growth and aging society. Hospital beds, as one of the key resources, may significantly affect the performance of a hospital system. Shortage of beds may have negative impact on the access to healthcare facility and patient safety, e.g., excessive waiting time before admission, overcrowded emergency room, cancellation of surgeries and unused operating theatre slots. Hence it is crucial for the healthcare service providers to manage the beds efficiently.

Bed management is a challenging task with many variations involved in different areas. In one aspect, the demand for beds could be highly fluctuating. The number of admissions per day varies by day of the week, e.g., there are usually more admissions during weekday than weekend ${ }^{[1]}$. Seasonal factors also contribute to the variation of daily admission, e.g., more admissions can be observed during flu seasons. A sudden surge of admission may lead to a temporary shortage of beds. Another major source of variation is the length of stay. The distribution of the length of stay is highly skewed by long stay patients, which affects the bed availability negatively. In addition to the variations existing in the practice of bed management, some policies may affect the availability of beds as well. For instance, it is a common practice to separate the beds into different classes in purpose of service differentiation, infection control, etc. Such a policy could cause the imbalance of resource utilization among different classes. Some classes are out of beds while some classes have surplus 
empty beds. All the above mentioned factors increase the complexity of bed management. Many research works have been done on different bed management strategies. Some of them focus on the admission part of bed management, e.g., smoothening the fluctuation of demand in beds by managing the elective and emergency admission more efficiently ${ }^{[2]}$, reducing the demand of beds by reforming patient flow, increasing the throughput and reducing readmission rates ${ }^{[3]}$. Some research works focus on the discharge part of bed management such as the root cause of delay in patient discharge ${ }^{[3]}$ and the benefits of early discharge ${ }^{[4]}$.

One key performance indicator many healthcare service providers are tracking in bed management is bed occupancy rate, which is measured by the ratio of occupied beds against the total number of beds in service. Bed occupancy rate is a direct reflection of bed utilization in a hospital. It was observed that shortage of beds was expected when the bed occupancy rate was over $90 \%{ }^{[5]}$. Higher bed occupancy rate is more likely cause higher emergency admission refusal rate ${ }^{[6]}$. A reliable prediction of bed occupancy rate within a certain time frame may help healthcare service providers better plan and utilize the limited bed resources.

There are a few research works focused on predicting the bed occupancy rate from a statistical perspective, e.g., forecasting of bed occupancy rate using trend fitting and time series analysis ${ }^{[7]}$. Phase type survival trees was applied to estimate the bed requirements ${ }^{[8]}$. One problem of above mentioned approaches is that the predicted results are based on the statistics of historical data, which may not reflect the variation of the latest situation.

Discrete event simulation is widely applied to many sections in healthcare systems such as outpatient clinic ${ }^{[9-12]}$, emergency department ${ }^{[13-15]}$, etc. A simulation model was constructed to predict the weekly bed occupancy rate ${ }^{[16]}$. Two inputs were needed for the simulation model: number of admission and length of stay. However, one problem of the above mentioned study is that the whole prediction procedure is offline. The number of admission and length of stay were generated from historical data using regression model and Poisson model respectively. All information of the prediction model is pre-defined and does not reflect the latest day-to-day variation. Thus it could be not accurate enough when the healthcare service providers want to know how the bed occupancy rate would looks like in the next few days.

The main contribution of this paper is to provide an online prediction procedure based on discrete event simulation. It can be used to predict the bed occupation rate in a short prediction horizon, e.g., the next week. Compared to the above mentioned research works, the method proposed in the paper is an online procedure with multiple stages. Within each stage, new information is collected and incorporated into the prediction procedure. The predicted bed occupancy rate is updated at each stage and reflects the variation of the latest situation. Such a prediction procedure is supposed to provide a more accurate projection for the decision making of the healthcare service providers, who are then able to plan properly and response quickly to the incoming bed situation.

\section{Methods}

\subsection{Framework of the prediction procedure}

Figure 1 illustrates the proposed prediction procedure. It includes $n+1$ stages. Stage 0 represents day 0 , the current situation. Stage 0 is the baseline of the whole projection. $O(0)$ denotes the number of patients occupying hospital beds in stage 0 , which is already known. Stage 1 represents day 1, the day next to day $0 . O(1)$ denotes the projected number of patients occupying hospital beds in stage $1, a(1)$ denotes the projected number of admissions in stage $1, d(1)$ denotes the projected number of discharges in stage 1. Similarly, stage $n$ represents day $n, O(n)$ denotes the projected number of patients occupying hospital beds in stage $n, a(n)$ denotes the projected number of admissions in stage $n, d(n)$ denotes the projected number of discharges in stage $n$. The detailed calculation is given as follows: 
$\mathrm{O}(1)$ can be calculated by Equation (1). $\mathrm{O}(\mathrm{n})$ can be calculated by Equation (2). $\mathrm{O}(\mathrm{n})$ can also be calculated using Equation (3) by combining Equation (1) and (2), where a(i) and d(i) denotes the number of admissions and discharges in stage i respectively.

$$
\begin{gathered}
O(1)=O(0)+a(1)-d(1) \\
O(n)=O(n-1)+a(n)-d(n) \\
O(n)=O(0)+\sum_{i=1}^{n} a(i)-\sum_{i=1}^{n} d(i)
\end{gathered}
$$

$d(i)$ can be considered as the sum of the past admissions which are discharged in stage i.d(i) can be calculated by Equation (4). Where $L(j)$ represents the projected distribution of length of stay in stage $j, P(L(j)=i-j)$ denotes the probability that the patient who is admitted in stage $j$ and discharged in stage $i$.

$$
d(i)=\sum_{j=-\infty}^{i-1} a(j) P(L(j)=i-j)
$$

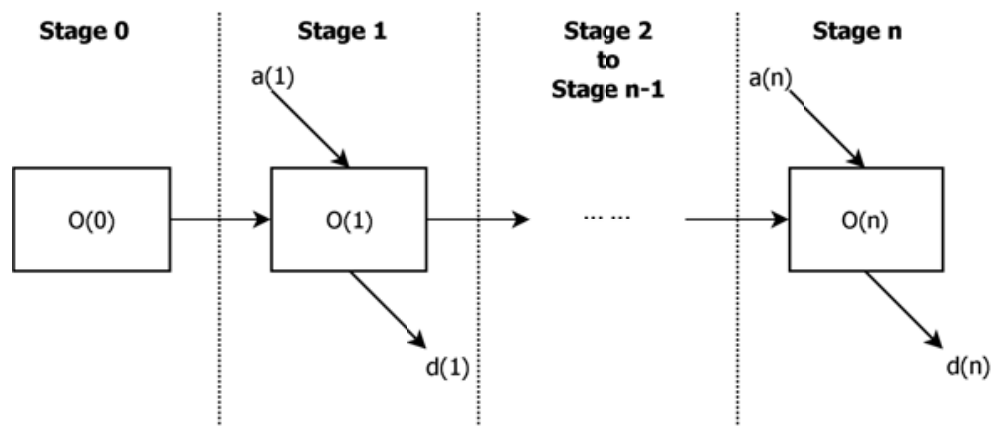

Figure 1. Framework of the prediction model

\subsection{Discrete event simulation model of the prediction procedure}

In this paper, a discrete event simulation (DES) model is constructed to predict the bed occupancy rate in the planning horizon by simulating the admission and discharge practice using Simul8 $2010^{[17]}$. The DES model takes three inputs: the number of beds in service, projected number of daily admission and expected length of stay of each admission. The output of the DES model is the performance indicator the healthcare service providers are interested, e.g., the bed occupancy rate within the planning horizon. The procedure is mainly composed of three steps:

Initialization. One specific day is selected as the initial stage (day 0). Usually the current day is selected in actual practice. The snapshot of the number of occupied beds is collected at the end of the day. The remaining length of stay of a specific patient is calculated using Equation (5). Where $L_{r}$ denotes the remaining of stay. $L^{\prime}$ denotes the re-estimated remaining length of stay. $L_{0}$ denotes the original estimation of length of stay when the patient is admitted. $t$ denotes the current date. $t_{0}$ denotes the admission date. $f$ denotes the update flag. When $f$ is 1 , the remaining length of stay is re-estimated by hospital staff during every initialization phase. When $f$ is 0 , the remaining length of stay is calculated based on the original estimation. In this paper, $f$ is set to be 0 .

$$
L_{r}= \begin{cases}L^{\prime} & \text { if } f=1 \\ \max \left(0, L_{0}-\left(t-t_{0}\right)\right) & \text { if } f=0\end{cases}
$$

Simulation. Starting from the initial stage, the DES model simulates the activities of patient admission and discharge till stage $n$, which is called prediction horizon in this paper. The number of admissions and length of stay at each stage follow a certain distribution derived from historical data. 
Calculation. Bed occupancy rate of each stage is calculated and returned as the simulation results.

The procedure described above is called one prediction cycle in this paper. As an online procedure, the whole prediction practice is continuously updated on the daily basis. There is one prediction cycle each day, which is initialized by the latest bed occupation status, remaining length of stay of the occupied beds and simulates till the next $n^{\text {th }}$ day. The subsequent prediction cycles follow the same practice with the updated information. In order to better capture the variations, there are multiple runs of the DES model in one prediction cycle and the summarized simulation results are returned.

\section{Results}

In this paper, the above mentioned procedure is applied to predict the bed occupancy rate of a ward in a Singapore general public hospital. The ward has 120 beds in total. One calendar year admission and discharge transaction data of 2011 is collected from hospital management information system. The transaction data is used to estimate the parameters needed for the simulation model. The simulation model is then used to predict the bed occupation rate of 2012. Prediction results are compared with the actual data to test the prediction accuracy. The table lists the distribution of the daily admission and length of stay derived from the transaction data. It is observed that the daily admission is significantly different between weekday and weekend/public holiday. The number of admission in weekend and public holiday is significantly less. Normal distribution is applied to fit the daily arrival. Gamma distribution is applied to fit the length of stay. The prediction horizon is set to be 10 days. Within each prediction cycle, the DES model runs 100 times, the simulation results are summarized in four terms: mean value $(\bar{R})$, standard deviation $(\sigma), 70 \%$ confidence interval $(70 \% C I)$ and $90 \%$ confidence interval $(90 \% C I)$.

Table. Distribution of the daily arrival and length of stay

\begin{tabular}{ll}
\hline Parameter & Distribution \\
\hline Weekday admission & $\mathrm{N}(24,2.8)^{*}$ \\
Weekend (including public holiday) admission & $\mathrm{N}(11,1.8)$ \\
Length of stay & $\mathrm{Gamma}(2.5,2)$ \\
\hline
\end{tabular}

${ }^{*} \mathrm{~N}$ denotes normal distribution;

Gamma denotes gamma distribution

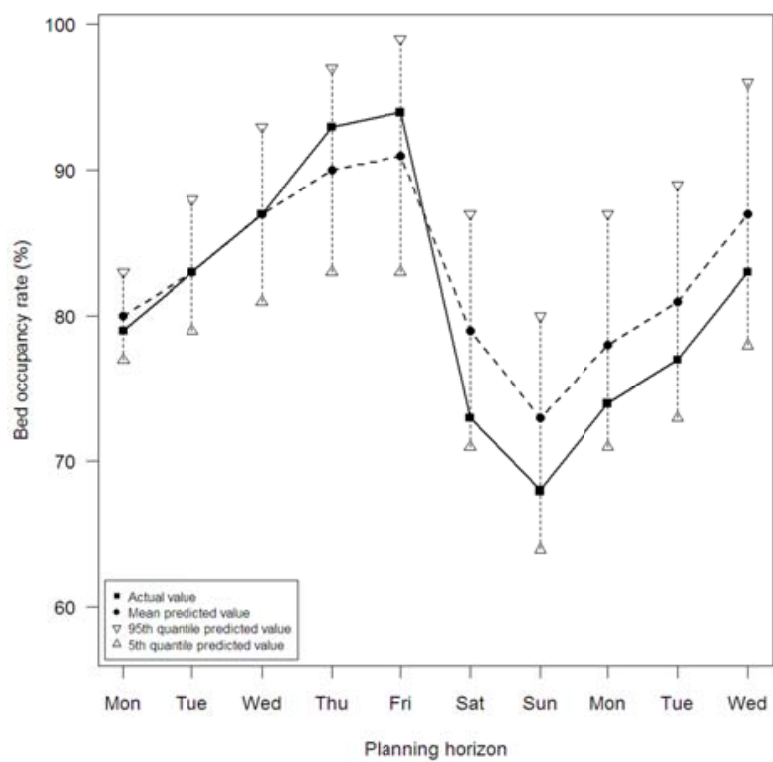

Figure 2. Simulation results of the prediction cycle 1

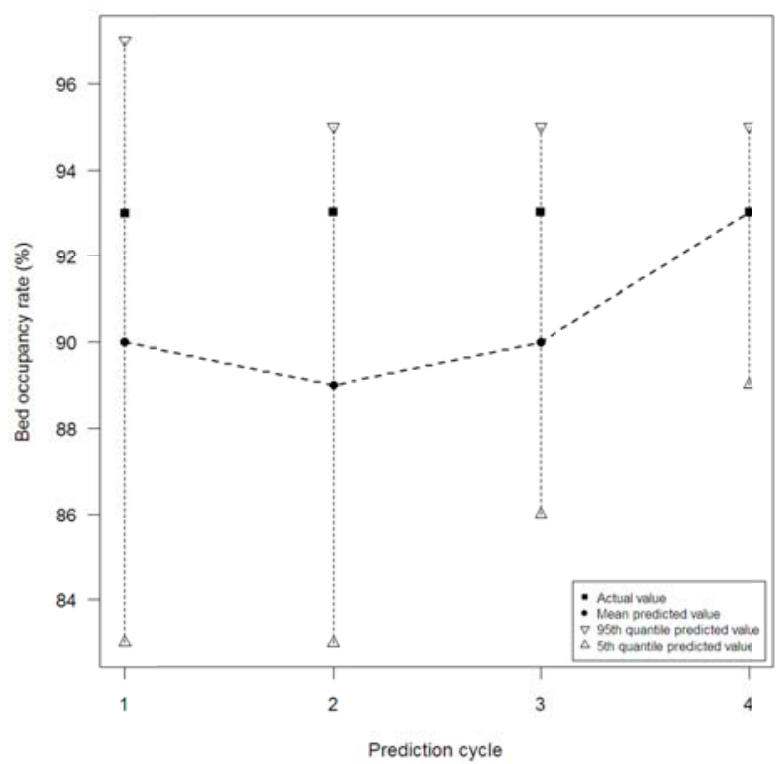

Figure 3. Comparison of the prediction value of a specific day (Thursday) in different prediction cycles 
Figure 2 illustrates the simulation results of one prediction cycle and the comparison with the actual bed occupancy rate $(R)$. Figure 3 illustrates the simulation results of a specific day (Thursday) in different prediction cycles.

\section{Discussion}

It is observed from Figure 2 that the simulation results match the actual values quite well in the earlier stages of the prediction horizon with smaller standard deviation and narrower confidence interval. The performance of simulation model deteriorates in the later stages with larger standard deviation and wider confidence interval. One possible explanation is that more sources of variations are injected into the prediction procedure when the stages increase. All the variations cause more fluctuating results.

It is observed from Figure 3 that the predicted value of the same day varies in different prediction cycles. The predicted value is better with a closer estimate and narrower confidence interval when the prediction is made within a near prediction cycle. Such a result indicates the strength of the online prediction procedure. The accuracy of the predicted values is continuously improving and closer to the actual values when more information is collected and fed into the prediction procedure. Hence the results from the online prediction procedure are more reliable compared to the offline ones.

\section{Conclusion}

Bed management is a challenging task due to the existence of various uncertainties. A proper prediction of the bed utilization for the next few days may help health service providers manage bed resources more efficiently. This paper proposed an online short-term bed occupancy rate prediction procedure based on discrete event simulation. Such a prediction procedure takes three inputs: number of beds, daily admission and length of stay. The whole prediction procedure is a continuous process composed of multiple prediction cycles. Each prediction cycle starts from an initial stage, simulates the admission and discharge practice within the prediction horizon and then returns the predicted bed occupancy rate of each day within the prediction horizon. Simulation results show that the predicted values are closer to the actual values in the earlier stage of the prediction horizon, and the predicted value of the same day is more accurate in a more recent prediction cycle, which indicates that the proposed prediction procedure performs better online than offline. One limitation of this study is that all the beds are considered generic and eligible to be occupied by any admissions. The complexity of partitions and transfer of cases among partitions are not considered. A bed occupancy rate prediction procedure for a complex bed partitioning system can be studied in the future research.

\section{Reference}

[1] N.C. Proudlove, K. Gordon, R. Boaden. Can good bed management solve the overcrowding in accident and emergency departments? Emerg Med J. 2003; 20: 149-155. PMid: 12642528. http://dx.doi.org/10.1136/emj.20.2.149

[2] I.A. Scott. Public hospital bed crisis: too few or too misused? AHR. 2003; 34(3): 317-324.

[3] A.F. Jennifer, I.M. Timothy, M. Marlene, S. Alan. Applying healthcare systems engineering methods to the patient discharge process. IJCENT. 2000; 1: 293-315.

[4] M. Barton, S. McClean, L. Garg, K. Fullerton. Modelling costs of bed occupancy and delayed discharge of post-stroke patients, 2010 IEEE Workshop on Health Care Management. 2010; 1-6. http://dx.doi.org/10.1109/WHCM.2010.5441244

[5] A. Bagust, M. Place, J.W. Posnett. Dynamics of bed use in accommodating emergency admissions: stochastic simulation model, BMJ. 1999; 319: 155-158. PMid: 10406748. http://dx.doi.org/10.1136/bmj.319.7203.155

[6] P.R. Harper, A.K. Shahani. Modelling for the planning and management of bed capacities in hospitals, J. Ope. Res. Soc. 2002; 53: 11-18. http://dx.doi.org/10.1057/palgrave/jors/2601278

[7] R.D. Farmer, J. Emami. Models for forecasting hospital bed requirements in the acute sector, J. Epidemiol. Community. Health. 1990; 44(4): 307-312. PMid: 2277253. http://dx.doi.org/10.1136/jech.44.4.307 
[8] L. Garg, S. McClean, M. Barton, B. Meenan, K. Fullerton. Forecasting hospital bed requirements and cost of care using phase type survival trees, Intelligent Systems (IS), 2010 5th IEEE International Conference. 2010; 185-190.

[9] P.R. Harper, H.M. Gamlin. Reduced outpatient waiting times with improved appointment scheduling: a simulation modelling approach, OR. Spec. 2003; 25(2): 207-222.

[10] K.J. Klassen, T.R. Rohleder. Scheduling outpatient appointments in a dynamic environment, J. Oper. Manage. 1993; 14(2): 83-101. http://dx.doi.org/10.1016/0272-6963(95)00044-5

[11] Z.C Zhu, B.H. Heng, K.L. Teow. Simulation study of the optimal appointment number for outpatient clinics, IJSIMM. 2009; 3: 156-165.

[12] Z.C. Zhu, B.H. Heng, K.L. Teow. Analysis of Factors Causing Long Patient Waiting Time and Clinic Overtime in Outpatient Clinics. J. Med. Sys. 2010; 36(2): 707-713. PMid: 20703659. http://dx.doi.org/10.1007/s10916-010-9538-4

[13] L.G. Connelly, A.E. Bair. Discrete event simulation of emergency department activity: a platform for system-level operations research, Acad. Emerg. Med. 2004; 11(11): 1177-1185. PMid: 15528582. http://dx.doi.org/10.1111/j.1553-2712.2004.tb00702.x

[14] S. Su, C.L. Shih CL. Modeling an emergency medical services system using computer simulation, Int. J. Med. Inform. 2003; 72: 57-72. PMid: 14644307. http://dx.doi.org/10.1016/j.ijmedinf.2003.08.003

[15] C.R. White, J.B. Best, C.K. Sage. Simulation of emergency medical service scheduling, Hosp. Top. 1992; 70: 34-37. PMid: 10119177. http://dx.doi.org/10.1080/00185868.1992.10543691

[16] A. Kumar, J. Mo. Models for Bed Occupancy Management of a Hospital in Singapore. Proceedings of the 2010 International Conference on Industrial Engineering and Operations Management. 2010; 1-6.

[17] Simul8 Corporation. Simul8, Available from: http://www.simul8.com. Accessed on 28 June 2012. 